\title{
To What Extent Do the Upbringing Context and Parenting Affect the Academic Performances of Students?
}

\author{
Tianqi Xue ${ }^{1 *}$ \\ ${ }^{1}$ University of Bristol \\ *Corresponding author.Email: bv20645@bristol.ac.uk
}

\begin{abstract}
Although the way to ensure the quality of education has always been a concern, the teaching quality does not entirely determine educational outcomes, and there are many reasons related to students themselves that need to be taken into account. In this way, the influencing factors of student achievement and education quality can be discussed in many aspects before policy changing. This paper mainly discusses the influence of students' growing environment and parenting, namely, external and internal context effects on academic achievement. By gathering, settling and analyzing secondary data, this article has came to a conclusion that the upbringing context and parenting affect the academic performances of students by multiple inner elements, and only to comprehensively develop both frameworks and components of the above does the attainment of pupils can be maximized.
\end{abstract}

Keywords: parenting, SES, parent personality, physical \& mental health

\section{INTRODUCTION}

Education is key in the 21 st century, where in most cases, social level, income, and opportunity are directly linked and determined by educational background. While, some of the pupils have a tricky access to education for example, nearly 1.6 billion children and youth were out of school and more than 5.5 million more girls were out of school than boys before the pandemic [1]. Others who actually have access to education are affected by multiple factors which makes it hard to ensure a sound academic achievement. These concrete facts illustrate that we are still far away from realizing the Quality Education goal, which if comes true can be a powerful method to upward social, economic mobility and escape poverty.

This paper aims to explore the connections and influences of parenting and upbringing context to students' academic performances. Then it explains how upbring environment and parenting affect students' education. This article would argue that the growth environment and parenting are vital factors that contribute to the academic grades of students, but their significance is built on comprehensive development rather than advantages of a specific domain.

\section{THE IMPACT OF GROWTH ENVIRONMENT ON STUDENT'S EDUCATION}

The upbringing environment in this article refers to external factors like social economic status of parents and social or cultural traditions other than inner elements like parenting style. These two elements affect students' education to a great extent.

\subsection{The social economic status of parents and family}

The upbringing environment in this article refers to external factors like community choices, peers, housing and social tradition other than inner elements like parenting style, family environment, and parent's anticipation or attitudes. The social economic status (SES) of parents can profoundly influence the formation and development of overall condition. However, it also has a complex history and definition. Capital used to be a condition for social scientists to measure socioeconomic status; owning to a family's human and social capital can easily affect happiness and development space, thus changing development outcomes [2]. 
SES has a tremendous impact on resources (nutrition, housing, and cognitive stimulation materials \& experiences) because SES has a direct link to nutrition, as low income cannot ensure sufficient food intake. According to Mortorell's model, inadequate dietary intake can lead to deficiencies in nutrient absorption, utilization, and resistance to infection. In turn, malnutrition leads to a range of morbidity and mortality [3]. Vulenzuela has stated that chronically malnourished children suffer from depression and other diseases that can reduce parental attention, support and sensitivity, leading to insecure attachment, stunted growth and other problems associated with a poor upbringing [4]. The housing is strongly linked with cognitive materials. In addition to leading to poor health and living environment, crowded housing is also related to the growth of students' cognition and emotional function. The researchers suggest that students from low socioeconomic status families are less likely to have access to entertainment and learning materials, which means they may have fewer opportunities for cognitive development and limited learning space at school. Because they do not have strong support from their families and parents [5]. For example, students with lower socioeconomic levels have fewer opportunities to participate in museums, libraries, and travel, so it is not easy to gain knowledge and motivation outside of school. As a result, in the absence of opportunities to fully communicate with society during self-development and growth, students may experience depression, declining performance, and even a series of behaviors that may antagonize parents and peers [3].

In terms of health, SES has a more significant impact on the external environment, such as medical conditions, lifestyle and parents' behavior, affecting students' physical and mental health development and academic performance. Students with low socioeconomic levels often lack health insurance and related safeguards, which reduces their access to medical care and can lead to manageable problems that can escalate into academic and health problems if left unchecked. Even in areas covered by medical insurance, low SES can cause psychological problems and affect students' academic development [6]. in addition, Bradley and Corwyn have mentioned that people with low SES live and work in an environment that is exposed to tobacco, alcohol, and drugs more frequently and have less exercise as well as diet control, which undoubtedly become an unsatisfactory role model and affect their children [3]. While their children tend to be hard to not being influenced by their peers who have experienced the same situation, thus early exposure to these harmful products and caught mental impact or problematic behavior.

SES influences expectation and style of parents on pupils, to be specific: parents' importance on students' independence, creativity, achievement, and language skills [7]. Parents of high socioeconomic status are likely to communicate with their children and pass on more experience to detect and control some destructive behaviors and thoughts in time. In contrast, low-SES parents are less likely to buy lots of books or arrange rich educational and cultural activities for their children. They tend not to neglect activities that may affect academic performance or physical health in the long run [5]. Meanwhile, teachers tend to see low-SES pupils less positively (their academic performance and selfregulatory abilities). These biases reduce the positive attention and reinforcement of good behavior teachers give to low socioeconomic status children [8]. It can be said that the teacher's attitude worsens the growing environment of the disadvantaged students in terms of learning materials and experience, which will undoubtedly strengthen the psychological resistance to learning and even behavioral problems. In a word, SES can be derived from multiple external factors such as housing, learning material, health, parental style, etc. And these elements together constitute the external growth environment of students and affect the family upbringing mode and physical and mental health of students.

\subsection{Cultural and social values}

The researchers shall not ignore that culture and social values significantly affect the external upbringing condition and parent's attitude to students. Shahidui and Karim have pointed out four reasons related to education quality and equality: Economic factors (parental investment \& schooling cost); Household factors (household works\& extend of involvement); Schoollevel factors (extracurricular activities, tutor gender, feminine facilities at school, and teacher's attitude); Cultural factors (cultural beliefs\&early marriage) [9].

There is a strong connection between these factors. For example, religion affects the educational balance to a large extent. Islam generally against education for girls. At the same time, these thoughts could be reflected in the construction of school facilities and the educational attitudes of teachers and parents towards male and female students, which leads to educational inequality. Moreover, the religious or cultural advocates of multiple children may also contribute to the economic problems and conflicts of the family, especially in economically underdeveloped areas, and change the children's education or aggravate the gender education level split. The research has shown that parental investments are vastly unfairly distributed between males and females under the influence of traditional social concepts, especially in less economically developed regions. Parents prefer to invest in their sons as males often carry on the responsibility to support parents later to be well cared for in the future. This preference causes them to spend most of their costs on the education of males rather than females when they are in short of money [10]. In rural China, for example, girls are more likely than boys 
to drop out of school when their parents cannot afford schooling fees [11].

Regarding households, Cultural attitudes have more influence on parents' decisions in the family. For instance, rural girls or firstborn are likely to start work at an early age. They always carry more responsibility to care for younger children and housework, affecting their school performance even leading to early school dropout [11]. These responsibilities are not only required by parents but also the moral code of society. However, at the school level, the culture and social value are mainly demonstrated in 2 aspects: attitudes to the gender of teachers and pupils and school hardware settings. Social values have primarily affected the construction and target people of school facilities. For example, most of the school facilities, such as basketball courts and soccer fields, are aimed at male students. In contrast, the poor prevalence of sports equipment for female students leads to low participation in extracurricular activities and reduces the sense of integration [9]. In addition, inadequate health facilities in some poor areas lead to long-term absence from school during menstruation, affecting academic performance.

Nevertheless, Some cultures contain social biases that reinforce gender bias in schools. For example, some teachers may believe that male students have more vital learning ability and development opportunities than female students. Thus, more inclined to help male students teach, resulting in differences in academic performance [9]. This attitude may inevitably stabilize the gender bias in some workplaces, causing adverse effects. In Islamic countries, the traditional belief supports that female students need to be taught by female teachers; therefore, many female students are forced to drop out because of the scarcity of female teachers in schools [12]. Religious and traditional beliefs are obstacles to education in most underdeveloped areas; girls are not allowed to go to school or obtain the same education and opportunities as men, which promotes a large number of early marriages and unequal education [13]. In India, the education of women is often seen as preparation for marriage, exacerbated by the persistence of religious ideas and the unequal orientation of government policies [14]. These in the economy, school, or family level appear the phenomenon of all reflect the particular region traditional ideas and social culture influence on students, education chance inequality and development are restricted by the conditions of the above, these concepts affect parents upbringing, ideas, and choices, limiting the environment and opportunities for development that education provides for female students.

\section{PARENTING STYLE}

Similarly, what affects parenting and parenting style except for the culture and social-economic level reasons also need to take into consideration to comprehensively understand the influence elements of parenting and its consequences to students.

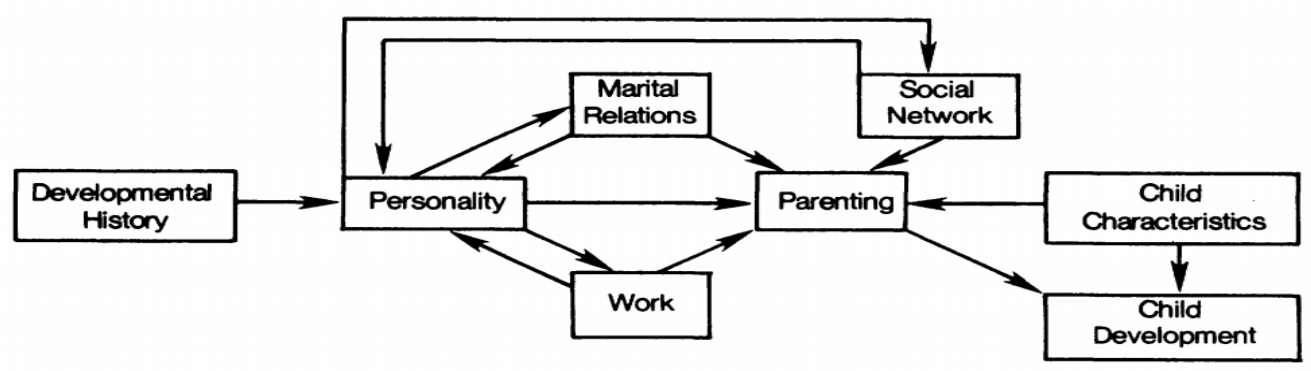

Figure 1. A process model of the determinants of parenting [15]

This figure shows the relationship and interactions between the different influence factors and parenting. According to Belsky, parenting is affected by ontogenetic origins, personal-psychological resources, contextual sources of stress and support, and a child's characteristics of individuality [15]. Because the parental function is multi-determined, any deficiency in the above factors can have an overall effect on the system. Of these, personalpsychological development is the most important factor, as it can have the most measurable impact on the physical and mental health of parents and their children [15]. Specifically, the development of individual psychology determines the methods and principles of dealing with people, thus affecting the growth experience, while experience indirectly changes social networks, marriage, and work. In turn, education, work experiences, and the emotional status of the couples also exert a subtle influence on the parents' mental health all the time, thus changing their parenting style and value [15]. for instance, favorable working conditions are good for parents' mental health, which makes them open and flexible in the way they deal with things or treat people, and vice versa. Stress and job uncertainty create adverse psychological reactions and often respond negatively to the demand of children, affecting the psychological well-being of them.

However, Lewis, Lerner and Lerner, claims the 'wellness of fit' among parents and children truly determines the parent-child relations, but neither the temperament and characteristics of the child nor that of parents [16]. This is because the differences between parents and children in personality, experiences, and other aspects are determined by a variety of external 
factors, so it can be said that the relationship between the two is constantly running in and out of the trial, rather than being determined unilaterally. As mentioned before, psychological or behavioral problems caused by parents' working conditions or income will also have an impact on parents' communication and management methods, thus changing children's personalities in many ways. Nevertheless, the difficulty of managing children's behavior and personality also affects the quantity and quality of parents' responses to their needs [17]. For example, parents tend to respond less to children who cry a lot, and regularly, naughty behavior is likely to get negative reactions.

Contextual sources of stress and support are also somehow connected with the social network, work, and marital relations owning to the fact that both could potentially promote or weaken parental functioning. The social supports (expectations, emotional, instrumental) that parents receive determine their psychological wellbeing, mental health, and functioning and reflect on methods and attitudes towards children [18]. Case in point, research demonstrates that Parents with less social support set more family rules and used more authoritarian methods of punishment [15]. In other words, the stress that parents experience in social life is directly transferred to the way they treat people and their children, thus causing indirect changes in the children themselves. So parenting is directly related to social support; children may be direct viewers of the consequences driven by psychological responses influenced by social support.

\section{DISCUSSION}

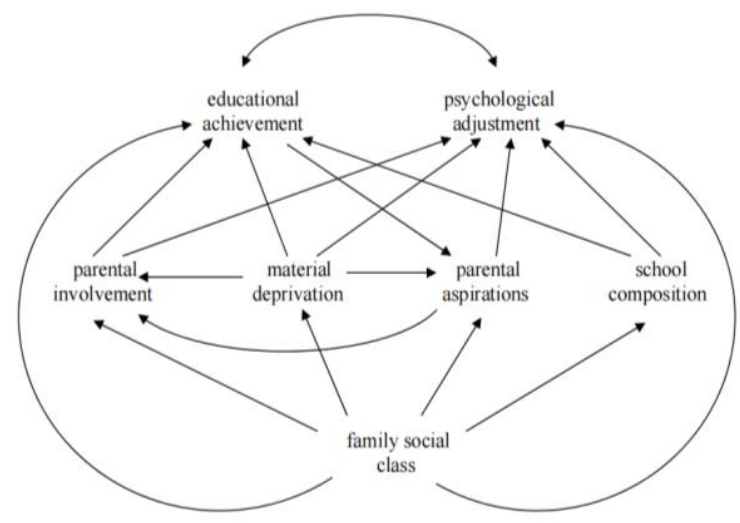

Figure 2. Sacker et al (2002) model of the relationship between family social class, and pupil achievement and adjustment

While establishing the influence of upbringing environment and parenting on academic performance, the author thought of the problems caused by SES discussed in the previous article. However, as deepening of the study, it is found that the influence of these two factors on academic performance is comprehensive, and the existence of each problem is mutually promoting and linked. Therefore, for settling the research questions mentioned in the introduction, it is necessary to explore the whole system of the connections among parenting, upbringing context, and students' academic achievement. The discussion part thus would primarily describe the connection between each of the influence factors and academic achievements to highlight the relationship within the structure of such matters. Overall, SES has a comprehensive impact on internal and external development and academic performance, while this model interprets the specific position of SES to a large extent. First of all, the resources and parental participation affected by SES are reflected in the figure as expectation, material deprivation, and participation, in which expectation changes participation.

In contrast, material deprivation affects the other two. Secondly, in terms of educational results, the above three factors interact, thus forming a cycle with the results. At the same time, SES itself also has a direct effect on the educational results. all three affect the psychological adjustment, while the educational achievement interacts with the it. In addition, SES acts a comprehensive relationship with health, while psychological adjustment ability, growth environment, nutrition, and upbringing style can predict and directly shape academic performance. As a factor with regional variables, social and cultural concepts have little to do with other reasons, but they still change the external environment and upbringing style. Although they have little relationship with other factors, on the whole, their influence on educational equity and achievement cannot be underestimated. Parents' experiences are related to childhood SES, guiding and shaping parents' character.

Furthermore, personality of parents shapes work, marriage, social networks, and parenting styles that affect future children's personalities. However, only when children's personalities and parents' parenting styles fit each other can a healthy family relationship and upbringing be created, thus positively promoting academic performance. For students' performance, the overall health level is the most significant factor, in which mental health is often related to stress response, SES and affects physical health. body health can also be affected by SES alone, which plays a role in the growth environment. The internal factors of parenting history also play a significant role in mental health. Therefore, academic performance is the fruit of education, and physical and mental health plays a role in the tree trunk.

Similarly, SES, parental character, and social thought should become the root of the tree. The overall result of development and cultivation is academic achievement, but its decisive factors are comprehensive rather than single, mutually affecting, and mutually beneficial rather than unilaterally determined. Therefore, the whole 
process affects the whole body, and any weakness will negatively impact the overall results.

\section{CONCLUSION}

This paper has explored the relationship between upbringing context and parenting and their influence on the academic performance of students by settling 2 of the research questions mentioned. By researching the effect of determine elements like SES, social and culture values and parenting on the academic performance of students and its relations to influence elements above, this paper holds and has proved that the comprehensive development of upbringing context and parenting is essential to realizing a high level of academic performance. With any of the weaknesses within these two factors, sound grades cannot be ensured.

\section{REFERENCES}

[1] United Nations, SDG Indicators, 2021. DOI: https://unstats.un.org/sdgs/report/2020/Goal-04/

[2] Krieger, N., Williams, D. and Moss, N., Measuring Social Class in US Public Health Research: Concepts, Methodologies, and Guidelines, Annual Review of Public Health, vol. 18(1), 1997, pp. 341378.

[3] Bradley, R. and Corwyn, R., Socioeconomic Status and Child Development, Annual Review of Psychology, vol. 53(1), 2002, pp. 371-399.

[4] White, K., The relation between socioeconomic status and academic achievement, Psychological Bulletin, vol. 91(3), 1982, pp. 461-481.

[5] Bradley, R., Corwyn, R., Burchinal, M., McAdoo, H. and Garcia Coll, C., The Home Environments of Children in the United States Part II: Relations with Behavioral Development through Age Thirteen, Child Development, vol. 72(6), 2001, pp. 1868-1886.

[6] MARMOT, M., Epidemiology of Socioeconomic Status and Health: Are Determinants Within Countries the Same as Between Countries? Annals of the New York Academy of Sciences, vol. 896(1), 1999, pp.16-29.

[7] Adams, B., The family, Fort Worth: Harcourt Brace College Publishers, 1995.

[8] McLoyd, V., Socioeconomic disadvantage and child development, American Psychologist, vol. 53(2), 1998, pp. 185-204.

[9] Shahidul, S. and Karim, A., Factors contributing to school dropout among the girls: A review of literature, European Journal of research and reflection in educational sciences, vol. 3(2), 2015.
[10] Leung, M. and Zhang, J., Gender preference, biased sex ratio, and parental investments in single-child households, Review of Economics of the Household, vol. 6(2), 2008, pp.91-110.

[11] Bledsoe, C., Fuller, B. and Liang, X., Critical perspectives on schooling and fertility in the developing world Which girls stay in school? The influence of family economy, social demands, and ethnicity in South Africa, Washington, D.C.: National Academy Press, 1999.

[12] Solotaroff, J., Afghanistan Gender Mainstreaming Implementation Note Series, No.1: The World Bank in south Asia, 2007. DOI: https://documents.worldbank.org/curated/en/96553 1467996720403/pdf/683660BRIOP1080LIC00TVE TOIN0English.pdf

[13] UNESCO, WORLD ATLAS of Gender Equality in Education, 2012, DOI: https://www.macfound.org/media/files/unescoworld-atlas-gender-education.pdf

[14] The PROBE Team, Public report on basic education in India, New Delhi: Oxford University Press, 1999.

[15] Belsky, J., The Determinants of Parenting: A Process Model, Child Development, vol. 55(1), 1984, pp. 83-96.

[16] Lewis, M., Lerner, R. and Lerner, J., Origins of Intelligence:Temperament intelligence reciprocities in early childhood: A contextual model, 2nd ed. New York, Springer, 2013.

[17] Campbell, S., Mother-infant interaction as a function of maternal ratings of temperament, Child Psychiatry \& Human Development, vol. 10(2), 1979, pp. 67-76.

[18] Mitchell, R. and Trickett, E., Task force report: Social networks as mediators of social support, Community Mental Health Journal, vol. 16(1), 1980, pp. 27-44. 